\title{
Comparison of CAV antibody titers in a vaccinated and naturally infected broiler breeder flocks
}

\author{
Payam Haghighi Khoshkhoo*, Gita Akbariazad and Mehrdad Tashakori \\ Department of Clinical Sciences, Karaj Branch, Islamic Azad University, Karaj, Iran. \\ Accepted 13 September, 2011
}

\begin{abstract}
The objective of the present work was to perform the efficacy of one attenuated live vaccine Nobilis CAV P4 (Intervet Co., Netherland) in broiler breeder and their progenies compared to naturally infected flock. The vaccine was administrated to Ross 308 broiler breeder at 6 week old through S.C. rout. A total of 352 serum samples were collected from vaccinated and unvaccinated breeder flocks (that infected naturally) from 6 to 33 weeks old and 2 times in their progenies at one-day old. Sera were analyzed using indirect Elisa kit (Synbiotic Corporation, USA) and data obtained were compared between 2 groups statistically. Due to natural infection of chicken infectious anemia (CIA) in unvaccinated breeder, mean titer in breeder and their progenies was significantly higher than vaccinated breeder and their progenies $(P<0.05)$. The CV\%s in breeders was not different significantly $(P>0.05)$ but $C V \%$ in progeny of unvaccinated breeder flock was twice more than progeny of vaccinated flock. There were 5 and $12 \%$ of zero titers of antibody in hens and progenies in unvaccinated breeder flock respectively. All breeders from vaccinated flock were positive serologically. It seems that vaccination could be an efficient rout for eliminating susceptible birds, decreasing variation in antibody titers and induction persistent and homogenous antibody titer in progeny.
\end{abstract}

Key words: Chicken infectious anemia (CIA), live vaccine, broiler breeder, efficacy.

\section{INTRODUCTION}

Chicken Infectious anemia $(\mathrm{CIA})$ is one of the most important immunosuppressive diseases in poultry. CIA virus belongs to the family Circoviridae which is without envelope and extremely resistant to physical and chemical agents. The virus spreads worldwide and it transfers vertically and horizontally (Schat and Van Santen, 2008). Due to high resistance of virus to disinfecting agents, it is near to impossible to eradicate it even in specific pathogen free (SPF) flocks (Fadly et al., 1994) and the only way to prevent the disease is either with natural infection or vaccination. Maternal antibody will remain in chicken sera for 3 weeks and will protect them against disease (McNulty et al., 1988; Jorgensen, 1990; Otaki et al., 1992).

Although the majority of breeder flocks will be infected naturally before breeding period, it seems that

${ }^{\star}$ Corresponding author. E-mail: pkhoshkho@kiau.ac.ir. Tel: +98 2614460465 . Fax: +982614482648. vaccination will be useful for controlled immunity. This field trial study was carried out to evaluate the efficacy of live CIAV vaccine in induction of immunity against the disease in broiler breeder flock compared with naturally infected flock and following up the titer transferred to their progenies. Also, we calculated and compared the numbers of sera with titer above 5000 in Elisa kit (Synbiotic Corporation, USA) in test and control groups based on the hypothesis of Canal et al who reported the relationship of VN titer and ELISA titer using the Synbiotic Elisa kit and found that Elisa titer above 5000 in breeders can protect their progenies against CIA in their first 4 week-old of age (Canal et al., 2004; Roussan, 2006).

\section{MATERIALS AND METHODS}

\section{Grouping}

Two commercial ROSS 308 broiler breeder flocks in different farms with the same age and situation were considered as test and control group with capacity of 5000 and 4000 chickens in each 
Table 1. AMT, CV\% of titers and titer $>5000$ in vaccinated breeder and their progenies flocks.

\begin{tabular}{|c|c|c|c|c|}
\hline $\begin{array}{l}\text { Indexes } \\
\text { Sampling time and flock }\end{array}$ & AMT & CV (\%) & $\begin{array}{c}\text { The percentage of titers }>5000 \\
(\%)\end{array}$ & $\%$ of zero titer \\
\hline Before Vac. $\left(\mathrm{B}^{\star}\right)$ & 3756 & 29.29 & 21 & - \\
\hline 5 weeks after vac. (B) & 5701 & 30.9 & 67 & - \\
\hline 3 months after vac. (B) & 7202 & 24.8 & 93 & - \\
\hline 4 months after vac. (B) & 6539 & 33.1 & 84 & - \\
\hline 5 months after vac. (B) & 6695 & 23.7 & 91 & - \\
\hline 5 months after vac. $\left(\mathrm{P}^{* *}\right)$ & 3438 & 17.8 & - & - \\
\hline 7 months after vac. $(P)$ & 3658 & 27.7 & - & - \\
\hline
\end{tabular}

${ }^{*} \mathrm{~B}$ : breeder, ${ }^{* *} \mathrm{P}$ : progeny.

Table 2. AMT, CV\% of titers and titer $>5000$ in unvaccinated breeder and their progenies flocks.

\begin{tabular}{lcccc}
\hline \multicolumn{1}{c}{ Indexes } & AMT & CV (\%) & The percentage of titers $>\mathbf{5 0 0 0}(\%)$ & \% of zero titer \\
\hline Sampling time and flock & 3010 & 27 & 18 & - \\
\hline Before Vac. $\left(B^{*}\right)$ & 6084 & 31.33 & 75 & - \\
5 weeks after vac. (B) & 9216 & 32.87 & 90 & 5 \\
3 months after vac. (B) & 9597 & 26.3 & 95.7 & - \\
4 months after vac. (B) & 9266 & 19.73 & 95.5 & - \\
5 months after vac. (B) & 5588 & 54.20 & 64 & 12 \\
5 months after vac. ( $\left.P^{\star *}\right)$ & 6039 & 33.88 & 69.6 & - \\
7 months after vac. (P) & & & & \\
\hline
\end{tabular}

${ }^{*} \mathrm{~B}$ : breeder, ${ }^{* *} \mathrm{P}$ : progeny.

group respectively. The growing and nutritional condition and vaccination schedule against other diseases were based on instruction for growing ROSS 308 broiler breeder and local status of health and disease.

\section{Vaccine administration}

One dose of live CIAV vaccine injected through S.C rout at 6 weekold of age in test group based on the recommendation of Company, whereas the control group did not receive the CAV vaccine. The live vaccine was Nobilis CAV P4 (Intervet Co., Netherland) each dose contained at least $3.0 \log _{10} \mathrm{TCID}_{50}$ of CAV strain 26 P4.

\section{Serology}

Blood sampling was randomly taken with $2 \mathrm{ml}$ syringe through wing vein in breeding flocks and through heart in progeny flocks. Serum samples were collected from vaccinated and unvaccinated flocks at the same times and the same numbers. Sera were taken in 6 period times in breeder from 6 to 33 weeks of age: before first vaccination, $5,12,16,20$ and 28th weeks after vaccination, and 2 times in their 1 day-old chicks at 20 and 28th weeks after vaccination in breeder. Number of serum in every sampling was 24 to 30 from each flock and totally 352 serum samples were collected and stored in $-20^{\circ} \mathrm{C}$ until the end of study. All samples were analyzed by Elisa kit (Synbiotic Corporation, USA) in the same condition. According to the kit's instruction, the serum samples with the S/P ratio equal or less than 0.349 were considered zero titer and S/P ratio equal or greater than 0.350 (titer equal or greater than 1472) were considered positive serum. Also, the titers above 5000 was compared between two flocks because it considered being protective against CIAV in their progenies based on previous studies (Canal et al., 2004; Roussan, 2006).

\section{Statistical analyses}

All data were analyzed and compared statistically by t-test using SPSS v13 software.

\section{RESULTS}

The two undesirable situations occurred during this field trial. First: despite several bloods sampling in breeder flocks before starting the trial, we could not find any broiler breeder flocks without antibodies against CAV, so, we had to do the trial in antibody-positive breeder flocks. In the other hand, according to serological data, the unvaccinated group was challenged naturally at age 8 to 9 week-old and the titer of antibody increased significantly. Arithmetic mean titer (AMT) and coefficient of variance (CV \%) in breeders flocks and their progenies in vaccinated and unvaccinated groups are given in Tables 1 and 2 respectively.

(1) Comparison of the AMT between two breeder flocks and their progenies showed that mean titers in unvaccinated group and their progenies due to the natural infection were significantly higher than vaccinated group 
and their progenies $(P<0.05)$. Antibody titer increased almost twice in vaccinated group after vaccination and three times in unvaccinated group after natural. Antibody titer reached at maximum level in vaccinated and unvaccinated breeder flocks 3 and 4 months after vaccination and natural infection respectively. Also, because of high antibody titration, the numbers and percentage of titers above 5000 in unvaccinated group was more than vaccinated group (75 vs. $93 \%$ ).

(2) Comparison of CV\% of titers in two groups showed that in breeder flocks, means CV\%s were 28 and 27.5 in vaccinated and unvaccinated breeder respectively which was not different significantly $(P>0.05)$. CV\% of progenies of unvaccinated breeder was twice more than progenies of vaccinated breeder (44 vs. 22.7) which was interesting finding.

(3) After vaccination, no zero titer was found in vaccinated breeder and their progenies, but in unvaccinated breeder flock and their progenies, 5 and $12 \%$ of serum samples showed zero titer respectively.

\section{DISCUSSION}

Based on the studies on prevalence of CIA in worldwide and Iran, it seems that most of the breeder flocks are antibody-positive against CIA, which can decrease the impotence and value of the vaccination against it (Jorgensen, 1990; Goodwin et al., 1990; Mahzounieh et al., 2005; Farhoodi et al., 2007). In this study, we could not find any anti-CAV negative-antibody breeder flocks, so we did the trial of efficacy of live Nobilis CAV P4 vaccine in positive-antibody breeder flocks. Besides of comparison of mean titer (AMT) and $\mathrm{CV} \% \mathrm{~s}$ in vaccinated and naturally infected groups and their progenies, we followed up the number of titers above 5000 with this kind of Elisa kit based on studies by Canal et al. (2004) who showed that the relationship of VN Elisa titer and reported that Elisa titer above 5000 in breeder by Elisa kit (Synbiotic Corporation, USA) can protect progeny against $\mathrm{CIA}$ in their life. They showed that in Brazil, in vaccinated breeder flocks $99 \%$ of hens had antibody titer $>5000$ and $1 \%$ had titer $4000-5000$, in contrast with unvaccinated breeder flocks that $52 \%$ had titer $>5000$ and $47 \%$ had titer $<5000$. Different studies show that vaccination can induce higher, more durable and more homogenous titer compared natural challenge in breeder flock which consequently results in safer immunity in progeny (Canal et al., 2004; Roussan, 2006). Progeny of vaccinated hens have sufficient levels of maternally-derived antibody to CAV for up to 3 to 4 weeks of age and after challenge haematocrit levels and weights of thymus in vaccinates remained within normal ranges whereas chicks from CAV non-vaccinated hens developed anemia and thymus atrophy following challenge (Otaki et al., 1992 and Pages-Mante et al., 1997). McNulty et al. (1988) in a longitudinal survey of two broiler breeder flocks demonstrated that maternal anti-CAA antibody persisted until about 3 weeks of age and both flocks began to seroconvert to CAA at 8 to 9 weeks of age. In this study, unvaccinated group was naturally infected at 8 to 9 weeks of age and so, the mean titer went up significantly higher than vaccinated group $(P<0.05)$ in breeder and their progenies. The $\mathrm{CV} \%$ was not different significantly between two breeder flocks $(P>0.05)$, but $C V \%$ of progenies of unvaccinated breeder flock was twice more than the progenies from vaccinated breeder flock $(44 \%$ compared to $22.7 \%$ ) which shows higher homogeneity of titer in vaccinated breeders and their progenies. The lowest and the highest titers in unvaccinated breeder flock were 0 and 15944 while in vaccinated breeder flocks, they were 2078 and 15161 respectively. However, $5 \%$ of unvaccinated breeder, in spite of natural infection, and $12 \%$ in their progenies remained negative-antibody which was an important finding. Zero titer in breeder flocks and consequently in their progenies will bring a dangerous situation for progeny especially at 3-4 weeks of age (Yuasa, 1994; Davidson et al., 2004). The durability of titer in vaccinated breeder flock decreased about $7 \%$ after 5 months. This rate in unvaccinated breeder flock naturally challenged was about $3 \%$. Antibody titer reached at maximum level in vaccinated and unvaccinated breeder flocks 3 months after vaccination and 4 months after infection respectively. Kapetanov et al. (2003) showed that the in unvaccinated highest mean titer breeder flock is at 18 weeks age, 12 weeks after natural infection. Vaccine increased the titer two times in vaccinated group compared to unvaccinated group which the titer increased three times after infection. In unvaccinated and vaccinated breeders, about 61 and $51 \%$ of maternally derived antibody (MDAB) transferred to their progenies respectively.

In conclusion, based on our results, the advantage of using live CIAV vaccine was the increase of titer and homogeneity of antibody titer in progenies of vaccinated breeder compared to naturally infected breeder. Besides, all progenies from vaccinated breeder had acceptable and protective level of $A B$. However, it seems that further complimentary studies are necessary to clarify the role of this vaccine more and to investigate the efficiency of vaccine in the yield of growing progeny.

\section{REFERENCES}

Canal CW, Ferreira DJ, Macagnan M, Fallavena LCB, Moraes HLS, Wald VB (2004). Prevalence of antibodies against chicken anaemia virus (CAV) in broiler breeders in Southern Brazil. Pesq. Vet. Brazil. 24: 89-92.

Davidson I, Shkoda I, Elkin N, Ayali G, Hamzani E, Kass N, Smith B, Borochovitch H, Gilat G, Krispin H, Kedem M, Perk S (2004). Chicken infectious anemia in young broiler flocks in Israel. Israel J. Vet. Med., 59: 78-82.

Fadly AM, Motta JV, Witter RL, Nordgren RM (1994) Epidemiology of chicken anemia virus in SPF chicken breeder flocks. Proceeding, International Symposium, Infectious Bursal Disease and Chicken Infectious Anemia, Germany. p 447. 
Farhoodi M, Toroghi R, Bassami MR, Kianizadeh M (2007) Chicken infectious anaemia virus infection among broiler chicken flocks in Iran. Arch. Razi Inst., 62: 1-6.

Goodwin MA, Brown J, Smeltzer MA, Cary CK, Girshick T, Miller SL, Dickson TG (1990) A survey for Chicken anemia agent antibodies in broiler breeders. Avian Dis., 34: 704- 708.

Jorgensen PH (1990) A micro scale serum neutralization test for the detection and titration of antibodies to chicken anemia agentprevalence of antibodies in Danish chickens. Avian Pathol., 19: 583593.

Kapetanov M, Orli D, Maja V, Slavica K, lazij S (2003) Clinical and serological examination of a parental flock latently infected with Chicken Anemia Virus. Acta Vet., 53: 239-248.

Mahzounieh M, Karimi V, Zahraei Salehi T (2005) Serologic evidence of Chicken Infectious Anemia in commercial chicken flocks in Shahrekord, Iran. Int. J. Poult. Sci., 4: 500-503.

McNulty MS, Connor TJ, Mcneilly F, Kirkpatrik KS, McFerran JB (1988). A serological survey of domestic poultry in the United Kingdom for antibody to chicken anemia agent. Avian Pathol., 17: 315-324.
Otaki Y, Saito K, Tajima M, Nomura Y (1992) Persistence of maternal antibody to chicken anaemia agent and its effect on the susceptibility of young chickens. Avian Pathol., 21: 147-151.

Pages-Mante A, Saubi N, Artigas C, Espuna E (1997) Experimental evaluation of an inactivated vaccine against chicken anaemia virus. Avian Pathol., 26: 721-729.

Roussan DA (2006) Serological survey on the prevalence of chicken infectious anemia virus in commercial broiler chicken flocks in Northern Jordan. Int. J. Poult. Sci., 5: 544-546.

Schat KA, Van Santen VL (2008) Chicken infectious anemia. In: Saif YM et al. (Eds.) Diseases of poultry. Ames: lowa State Press, pp 100-110.

Yuasa N (1994) Pathology and pathogenesis of chicken anemia virus infection. Proceeding International Symposium Infectious Bursal Disease and Chicken Infectious Anemia, Germany. p. 385. 